\title{
Quantification of defects in an aluminum plate using direction- tunable shear horizontal wave imaging
}

\author{
Mubarak Ahmad ${ }^{1,2}$, Zenghua Liu ${ }^{1,2^{*}}$, Abdul Basit ${ }^{1,2}$, Wenshuo Jiang ${ }^{1,2}$, Wu Bin ${ }^{1,2}$, Cunfu He ${ }^{1,2}$ \\ ${ }^{1}$ Faculty of Materials and Manufacturing, Beijing University of Technology, Beijing 100124, China \\ ${ }^{2}$ Beijing Engineering Research Center of Precision Measurement Technology and Instruments, Beijing University of Technology, \\ Beijing 100124, China
}

\begin{abstract}
The quantification of damage, in plates, pipes and such like structures, is one of the current research areas. In this study, to estimate the notch size, shape, and orientation was carried out experimentally based on fundamental shear horizontal mode, $\mathrm{SH}_{0}$ mode. Using least number of transmitters, the reconstruction algorithm for the probabilistic inspection of damage (RAPID) has been used to carry out fast and efficient investigation of scattered waves using $\mathrm{SH}_{0}$ wave interaction with notch at various incident angles. An approach of ultrasonic guided waves and (RAPID) algorithm, using a direction-tunable shearhorizontal mode array magnetostrictive patch transducer (DT-SHMA-MPT), the notch in a thin aluminum plate is experimented. Firstly, the RAPID algorithm, based on $\mathrm{SH}_{0}$ mode, is used here to quantitatively study a notch in a thin aluminium plate. Secondly, the imaging application using DT-SHMA-MPT is achieved. Experiments were conducted using an array of eight DT-SHMA-MPTs based on a pitch-catch mode to utilize the scattered waves at the notch. The results obtained through experiments reveal the effect of directionality of the scattered waves and have find out the optimal transducers configuration. The localization, quantification and orientation at the same time are obtained using only two or three transmitters. To improve the accuracy of imaging, a threshold value was selected. The presented approach can be used to detect, locate and image the surface defects in aluminum plate.
\end{abstract}

\section{Introduction}

For the inspection of plate and plate like structures, shear-horizontal ( $\mathrm{SH})$ guided wave is one of the basic methods, where the fundamental $\mathrm{SH}_{0}$ mode is widely used in various fields [1-5]. Due to its non-dispersive nature and constant group velocity, the $\mathrm{SH}_{0}$ mode has several advantages in the field of non-destructive testing [6-9]. To improve the coupling efficiency of $\mathrm{SH}_{0}$ mode using electromagnetic acoustic transducer (EMAT), Ogi et. Al [10] has varied the incident angle of the bias magnetic field based on magnetostriction. The phenomena of magnetostriction has a magnetization process and a change in dimension of ferromagnetic materials, e.g, iron, nickel, and cobalt [11]. Due to applied external field, a ferromagnetic material may experience a change in its dimension. This dimensionality change is known as joule effect [12]. As a magnetic field is required for the generation and reception of ultrasonic waves in the inspecting material, hence it is often required as a kind of EMAT. Using a ferromagnetic material, magnetostriction and Lorentz force both are generated in the structure when a magnetic field is applied, where the former is dominant phenomena. Using magnetostriction for the generation and reception of guided waves [13-15], great progress has been achieved to develop a magnetostrictive transducer for the applications of nondestructive testing. For the inspection of non-ferromagnetic material or having a high transduction efficiency, nickel patches can be employed for magnetostrictive patch transducers (MPTs) [16]. For the applications of plate, MPTs can be used to excite directional [17-21] as well as omnidirectional $\mathrm{SH}_{0}$ mode $[22,23]$. Recently, many researchers have briefly reported the work on the interaction of guided waves with defects in regular structures, like, plates and pipes [24-33]. In an isotropic plate the scattered and angular influence of $\mathrm{SH}_{0}$ mode for through thickness crack and partial thickness transverse crack is investigated [34-36]. The interaction and scattering of $\mathrm{SH}_{0}$ mode with longitudinal cracks in plates was also studied and presented [38]. Different types of defects, such as, cracks that can be perpendicular or parallel to the weld were investigated [37]. $\mathrm{SH}_{0}$ mode can be easily excited in a plate using EMAT [39]. To generate directional $\mathrm{SH}_{0}$ mode in a thin plate, a periodic permanent magnet (PPM) and an axially wound coil is used [40]. An EMAT including a meander line coil and static bias magnetic field is developed for the generation of SH wave [41]. Similarly, based on magnetostriction the directed $\mathrm{SH}_{0}$ mode can be generated with the use of a figure-of eight coil to change the angle between coil and magnet [15].

This study investigates the quantification, imaging and localization of a notch using experimental data based on imaging methods. A RAPID algorithm was used for the fast and efficient imaging of notch. Based on this algorithm, two fusion approaches were deployed which

\footnotetext{
* Corresponding author: liuzenghua@bjut.edu.cn
} 
are RAPID-based full summation and RAPID-based full multiplication. Each approach was investigated based on the scattered waves using the pitch-catch experimental mode. Imaging results are further obtained using modified methods to eliminate the influence of uneven probability distribution of the array (UPDA) in RAPID algorithm. This work has a direction-tunable shearhorizontal mode array magnetostrictive patch transducer (DT-SHMA-MPT) for the inspection of aluminum plate [42]. The DT-SHMA-MPT is neither a directional transducer nor an omnidirectional transducer due to its smaller angle of direction than omnidirectional transducer and larger angle of direction than directional transducer. The DT-SHMA-MPT detects the signal with a wider range as compared to the directional transducer.

\section{RAPID imaging algorithm}

Based on RAPID algorithm, the inspected area is well defined and the probability of defect at each point is calculated. Using only a pair of transducer, it is not possible to image an object accurately. Hence, an array of transducers is being designed and deployed to accurately extract an image. To extract an image, using received signals collected through an array of transducers, image fusion approaches are used. Here, two basic fusion approaches are used. One is the RAPID-based full summation imaging approach, which is illustrated as [43]:

$$
P(x, y)=\sum_{i=1}^{N} \sum_{j=1, i \neq j}^{N} P_{i j}(x, y)=\sum_{i=1}^{N} \sum_{j=1, i \neq j}^{N} A_{i j} \frac{\beta+1-R_{i j}(x, y)}{\beta}
$$

where $P_{i j}(x, y)$ is the probability of damage at a point ( $x$, $y$ ) in the defined scanning area, $N$ is the number of transducers, and $i$ describes the transmitter whereas $j$ describes the receiver. Similarly, $\beta$ shows the shape factor. Whereas $R_{i j}(x, y)$ is defined as:

$$
R_{i j}(x, y)=\left\{\begin{array}{cc}
R D_{i j}(x, y), & R D_{i j}(x, y) \leq \beta+1 \\
\beta+1, & R D_{i j}(x, y)>\beta+1
\end{array}\right.
$$

and

$$
R D_{i j}(x, y)=\frac{\sqrt{\left(x_{i}-x\right)^{2}+\left(y_{i}-y\right)^{2}}+\sqrt{\left(x_{j}-x\right)^{2}+\left(y_{j}-y\right)^{2}}}{\sqrt{\left(x_{i}-x_{j}\right)^{2}+\left(y_{i}-y_{j}\right)^{2}}}
$$

The value of $A_{i j}$ defines a signal difference coefficient (SDC), which is the difference of a reference/base line signal (signal without damage) and damage signal. SDC is defined as:

$$
S D C=1-\frac{C_{D B}}{\sigma_{D} \sigma_{B}}=\frac{\sum_{t=0}^{t_{a}}\left[D(t)-\mu_{D(t)}\right]\left[B(t)-\mu_{B(t)}\right]}{\sqrt{\sum_{t=0}^{t_{a}}\left[D(t)-\mu_{D(t)}\right]^{2} \sum_{t=0}^{t_{a}}\left[B(t)-\mu_{B(t)}\right]^{2}}}
$$

where $\mu_{D(t)}$ and $\mu_{B(t)}$ are known as standard deviation of $D(t)$ and $B(t)$, respectively, whereas $\sigma_{D}$ and $\sigma_{B}$ show the mean values of $D(t)$ and $B(t)$, respectively, $D(t)$ presents damage signal, $B(t)$ presents reference signal, $C_{D B}$ is the covariance of $D(t)$ and $B(t)$. High value of SDC shows large difference in $D(t)$ and $B(t)$.

Another fusion approach is RAPID-based full multiplication, which is given as [44]:

$$
Q(x, y)=\prod_{i=1}^{N} \prod_{j=1, i \neq j}^{N} Q_{i j}(x, y)=\prod_{i=1}^{N} \prod_{j=1, i \neq j}^{N} A_{i j} \frac{\beta+1-R_{i j}(x, y)}{\beta}+1
$$

where $Q(x, y)$ is the probability of damage at a point ( $x$, $y)$. Each transducer pair $i j$ has its unique detection path. After obtaining the SDC values from all transducer pairs, the RAPID algorithm is used to reconstruct an image. The extracted image is dependent on the spatial distribution of each SDC value. The distribution of the ray path is described in Fig. 1. Where a point $\left(x_{i}, y_{i}\right)$ shows the transmitter position and $\left(x_{i}, y_{i}\right)$ shows receiver position. This area depends on the parameter $\beta$, where the amplitude is maximum at the center line and away from the center line its value decreases as compared to its center. This parameter $\beta$ is known as the shape factor, to control the size of the ellipse.

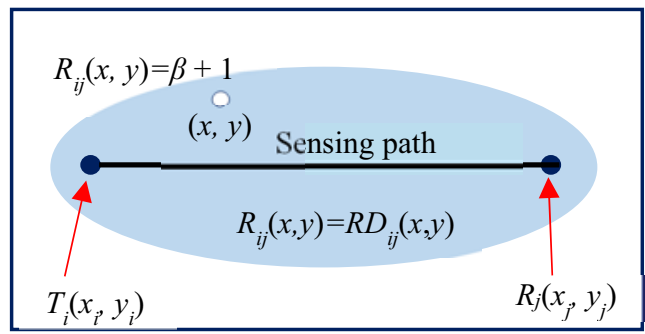

Figure 1. Distribution of wave signal between a pair of transducer

\section{DT-SHMA-MPT}

Based on the mechanism of magnetostriction, to generate and receive $\mathrm{SH}_{0}$ mode in an aluminium plate, a DTSHMA-MPT we developed [42] is shown in Fig. 2.

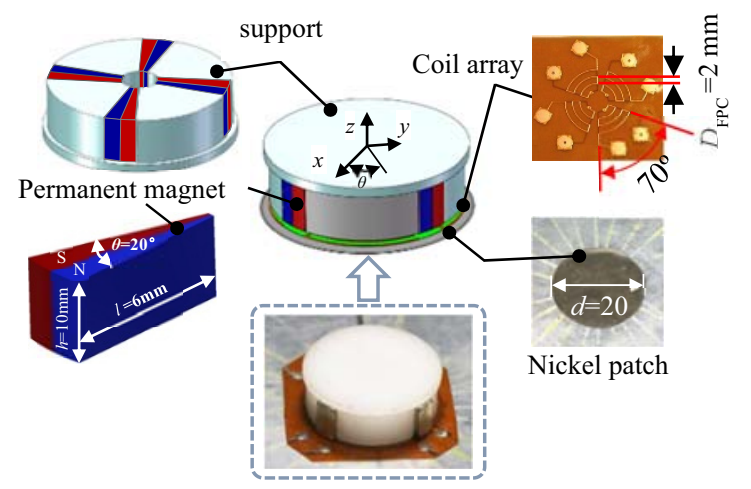

Figure 2. Configuration of DT-SHMA-MPT

DT-SHMA-MPT consists of a number of fan-shaped permanent magnets, a coil array, a piece of nickel patch, and supporting sleeves [42]. A nickel patch, in a circular shape, is glued at the surface of the inspecting plate using an adhesive epoxy. The white plastic nylon 
material is used as a supporting sleeve. The sleeves are of fan-shaped spaces, arranged in a symmetric pattern. The circumferentially polarized permanent magnets are being designed and manufactured. The height $h$ of each permanent magnet is $10 \mathrm{~mm}$, the radial length 1 is $6 \mathrm{~mm}$, and the central angle $\theta$ is $20^{\circ}$. The polarity of two sides of angle bisector are $N$ pole and $S$ pole. The coil array is designed as a thin flexible printed circuit (FPC). It has a centrally symmetric fan-shaped meander coil, with an angle of $70^{\circ}$. Each coil has a double-layer structure, presented in the inset of Fig. 2. Both the layers have a series connection. The spacing, $D_{\mathrm{FPC}}=2 \mathrm{~mm}$, of the wires in the coil is based on the central frequency, theoretically calculated using dispersion curves. The distribution of acoustic field for the proposed DTSHMA-MPT was experimentally investigated. The dependency of acoustic field direction to the circumferential static magnetic flux density, $B_{\text {s-phi, }}$ on the aluminum plate was tested and analyzed. In experiments, the excitation transducer was remained unchanged whereas the receiving transducers were given a rotation around z-axis counter clockwise.

\section{Experimental investigation of notch}

\subsection{Experimental setup}

The schematic diagram of experimental setup using an array of eight DT-SHMA-MPTs is shown in Fig. 3. An aluminum plate having a size of $1000 \mathrm{~mm} \times 1000 \mathrm{~mm} \times$ $1 \mathrm{~mm}$ is used as a testing material. A $600 \mathrm{~mm} \times 600 \mathrm{~mm}$ area was chosen for the scanning. The dimension of the notch is given as: length $=16 \mathrm{~mm}$ and width $=0.5 \mathrm{~mm}$. The orientation of the notch is illustrated in Fig. 3. Taking the left bottom corner as an origin, the center of the horizontal notch was located at $(300,300)$.

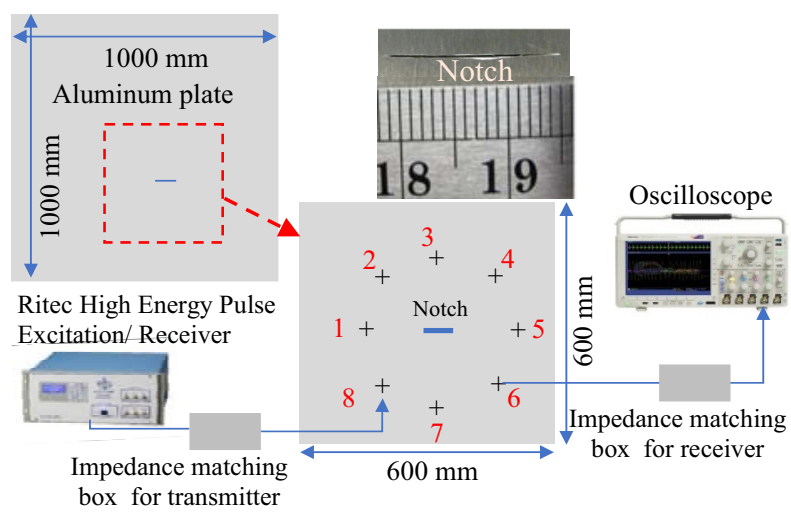

Figure 3. Experimental setup of using the transducer array for notch detection

The transducer array is localized in a circular pattern around the notch. The array has eight DT-SHMA-MPTs, deployed for the generation and reception of $\mathrm{SH}_{0}$ mode in the aluminum plate through pitch-catch mode. The diameter of the circular array is $500 \mathrm{~mm}$. For the improved and efficient energy conversion, a pair of impedance matching array is used to connect with the excitation transducer and receiver transducer. An excitation signal of, 5-cycle sinusoidal signal with a center frequency of $782 \mathrm{kHz}$ modified by Hanning window, is provided using a power amplifier RPR-4000 which can also amplify the received signal. The multichannel digital oscilloscope is used to observe and store the received signal. In the experiments, the imaging was done for the localization, sizing and orientation.

\subsection{Scattered waveforms for the pitch-catch mode}

Firstly, the waveform results of the pitch-catch experiment are presented. For the sake of simplicity, the signals excited by transmitter, No. 3, and received by remaining receivers are only presented as shown in Fig. 4. In this configuration, transducer No. 3 was the transmitter and the remaining seven transducers were used as receivers.

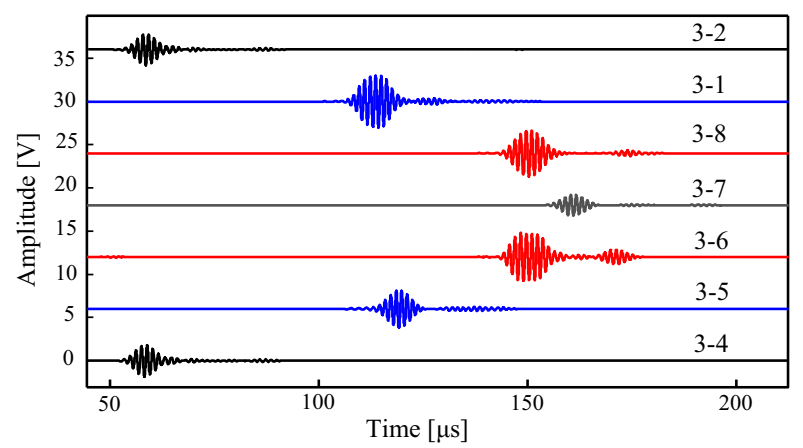

(a)

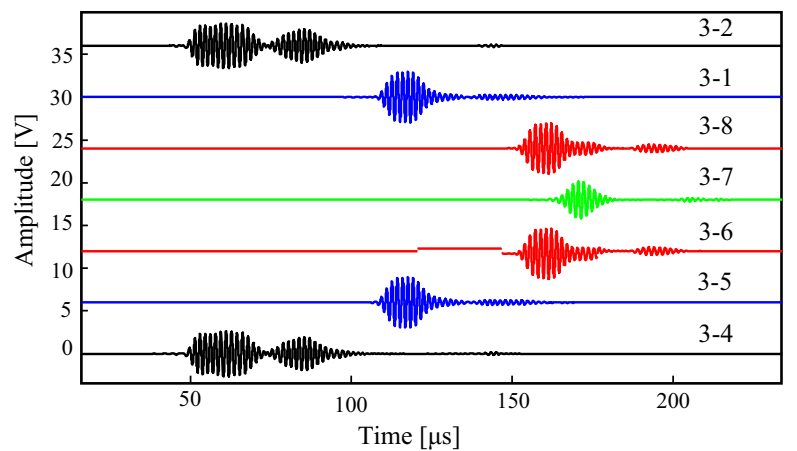

(b)

Figure 4. The waveform signals in pitch-catch experimental mode: (a) baseline signals without any notch and (b) signals with 16-mm notch (DT-SHMA-MPT No. 3 as a transmitter and the rest of the seven DT-SHMA-MPT transducers as receivers)

Fig. 4(a) presents baseline signals, whereas Fig. 4(b) shows a number of received signals having a $16-\mathrm{mm}$ notch. The received scattered waveforms for the incident $\mathrm{SH}_{0}$ mode (transmitter $\mathrm{T}_{3}$ ) are illustrated in Figs. 4(a) and 4(b). The strongest received signal was recorded by the nearby transducers. In both the figures, the first wave packet is the received $\mathrm{SH}_{0}$ mode. In Fig.4(a), there is no defect. So the signals have no scattering. Whereas in Fig.4(b), due to the notch, scattered waves exist near the first wave packet. These scattered waves are some other wave modes that may exist after the interaction of $\mathrm{SH}_{0}$ mode with the edges of the notch. If we subtract the baseline signals from received signals through defect, 
there would be some useful information present about the defect. For every transmitter, there exists seven possible paths to record the wave signals at the receivers. There also exists some further scattered wave packets because of the notch in the specimen. To obtain the scattered wave signals, different experiments have been conducted for all possible combinations of transmitter and receivers. The imaging algorithm was then used for the quantification of defect based on these received signals.

\section{Results}

The results obtained are significantly dependent on the direction of incident wave with respect to the notch. The notch localization and imaging results using, RAPIDbased summation and RAPID-based multiplication approaches, for transmitters are presented in Fig. 5. The notch location in the image is indicated by the pixel with the highest field value (the brightest pixel). Three different directions are used for the incident $\mathrm{SH}_{0}$ mode. When the incident wave propagates at $0^{\circ}$ to the notch, the imaging result is very weak since the brightest pixel seems near the transmitter, as shown in Fig. 5(a). This is due to the very weak scattered signals at $0^{\circ}$ incidence. Similarly at $45^{\circ}$ incident $\mathrm{SH}_{0}$ mode, the imaging result, as shown in Fig. 5(b) has no such difference because the scattered signals are relatively changed in a very little proportion. Lastly, at $90^{\circ}$ incidence of the $\mathrm{SH}_{0}$ mode (the incident wave was perpendicular to the notch orientation), the imaging result, presented in Fig. 5(c), is very good because of the strongest scattered signals among these three cases.

So it could be summarized that to estimate the notch size one transmitter may not be adequate, but it may expect the location of the notch if it is located in a suitable location with respect to the notch. For the estimation of notch size, based on RAPID-based full summation imaging and RAPID-based full multiplication, imaging of the notch was estimated using a pair of transmitters (at a time for each transmitter, the rest of the seven DT-SHMA-MPTs were the receivers). The imaging results obtained through this configuration are illustrated in Fig. 6.
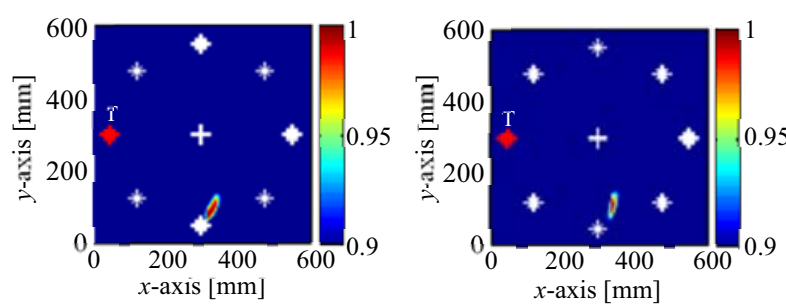

RAPID-based full summation

RAPID-based full multiplication

(a) $0^{\circ}$ incident $\mathrm{SH}_{0}$ wave
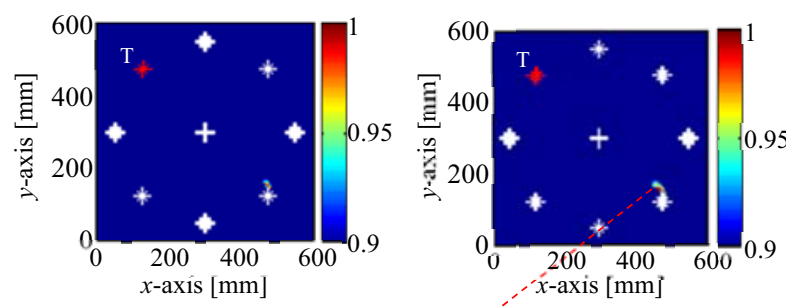

RAPID-based full summation

RAPID́-based full multiplication

Center of actual notch $(300,300)$

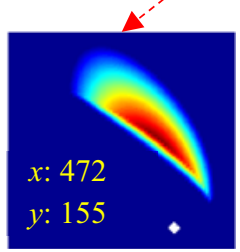

Zoomed in damage

(b) $45^{\circ}$ incident $\mathrm{SH}_{0}$ wave
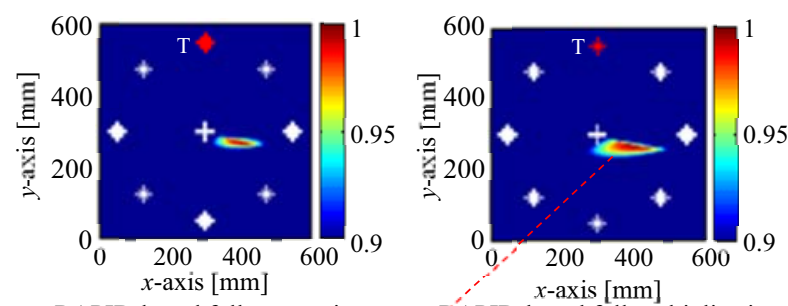

RAPID-based full summation RAPID-based full multiplication

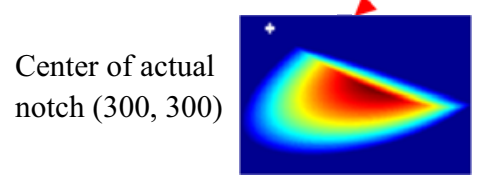

Zoomed in

damage

(c) $90^{\circ}$ incident $\mathrm{SH}_{0}$ wave

Figure 5. Directionally effect on the imaging results of RAPID-based full summation imaging and RAPID-based full multiplication at (a) $0^{\circ}$ incident, (b) $45^{\circ}$ incident, and (c) $90^{\circ}$ incident.

In Fig. 6(a), two transmitters, e.g, No. 1 and No. 3 are used once at a time and remaining transducers were used as receivers. The received signals at all the receivers for each transmitter were recorded and analyzed. Using these received signals the image was obtained. Both the methods have quantified and located the notch efficiently with the slight change in the orientation of the notch. As compared to the original length of $16 \mathrm{~mm}$ of notch, the notch obtained through method 1 and method 2 is $23 \mathrm{~mm}$ which resembles to the original notch dimension, similarly the location is $(325$, $348)$ as compared to $(300,300)$ which is not good. 


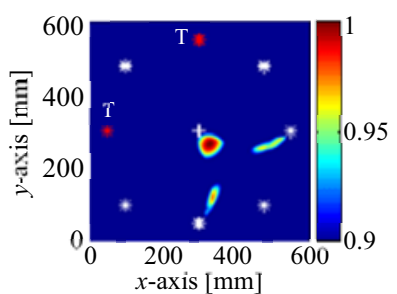

RAPID-based full summation
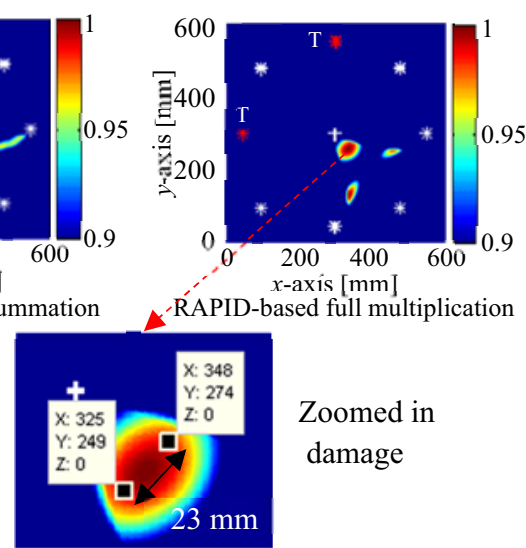

Zoomed in

damage

(a) DT-SHMA-MPTs No. 1 and No. 3

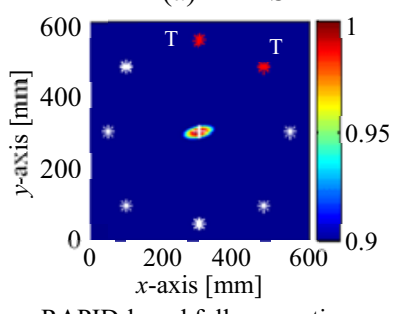

RAPID-based full summation

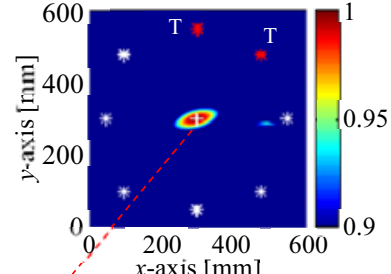

RÁPID-based full multiplication

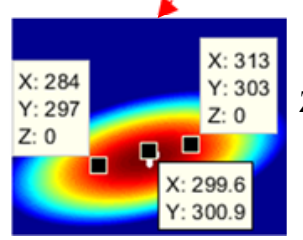

Zoomed in damage

(b) DT-SHMA-MPTs No. 3 and No. 4

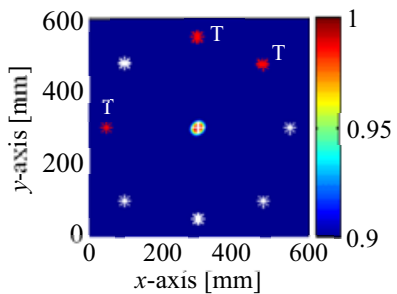

RAPID-based full summation

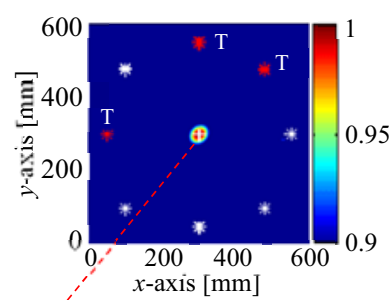

RAPID-based full multiplication

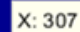

$\mathrm{Y}: 306$ Y. 306

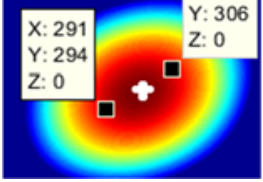

Zoomed in

damage

(c) DT-SHMA-MPTs No. 1, No. 3 and No. 4

Figure 6. Estimation of notch size based on method 1 and method 2 imaging results using the sensing paths of two transmitters (a) DT-SHMA-MPTs No. 1 and No. 3, (b) DTSHMA-MPTs No. 3 and No. 4, (c) DT-SHMA-MPTs No. 1 , No. 3 and No. 4.

In Fig. 6(b), the transducers No. 3 and No. 4 are used as transmitters and remaining transducers were used as receivers. Using the obtained received signals the image was extracted. Both the methods have located the notch efficiently at $(300,300)$ exactly at the required location with good orientation of the notch with obtained size of $29 \mathrm{~mm}$ which is larger than the original. Similarly, in Fig. 6(c), this time three transducers No. 1, No. 3 and No. 4

are used as transmitters and remaining transducers were used as receivers. Using the received signals the image was extracted. Both the methods have located the notch very accurately with good orientation of the notch and same size of $16 \mathrm{~mm}$ as shown in enlarged image. From the experiments and experience of using these DTSHMA transducers, we can summarize that the above positions of transducers can be used only for the proposed transducers. Besides these three above cases, shown in Fig. 6, there would be no imaging of notch.

Using Fig. 6, we can conclude that to image a notch in plate the best positions of transmitters (considering only two or three transmitters instead of whole array of transducers) are at the positions No. 1, No. 3 and No. 4. Two points with a maximum probability can be observed, representing the two tips of the notch. A threshold value (about $80 \%$ of highest field value) is chosen for the imaging results as shown in Fig. 6. The two tips of notch shown in zoomed-in image can be identified.

\section{Conclusion}

The principles of imaging methods have been explained briefly in this paper. Experiments were performed at different incident angles of $\left(0^{\circ}, 45^{\circ}\right.$, and $\left.90^{\circ}\right)$ to the notch using $\mathrm{SH}_{0}$ mode. The influence of imaging results based on different directions were studied. For the experiment, a notch was designed and manufactured in the plate. To quantify the notch size using the imaging methods, the experiment has an array of eight transducers using a pitch-catch mode. The proposed methodology has provided a fast and efficient investigation of notch location, length and orientation. Both the incident wave and sensing directions of $\mathrm{SH}_{0}$ mode effect the scattered wave signals through the notch, which finally effect the quality of imaging results. The experiments show that, both the imaging methods can be used successfully to quantify the notch size, its localization, and orientation. The suitable transducer configuration may result in accurate defect quantification.

\section{Acknowledgment}

The National Key R\&D Program of China (No. 2018YFC1902405) and the National Natural Science Foundation of China (Nos. 11772014 and 11527801) supported this study.

\section{References}

1. P.A. Petcher, S. Dixon, NDT\&E Int, 74, 58-65, (2015)

2. H.M. Seung, C. Park, Y.Y. Kim, Ultrasonics, 69, 58-66, (2016)

3. Zhao X, J.L. Rose, J Acoust Soc Am, 115, 19121916, (2004)

4. S. Hill, S. Dixon, NDT\&E Int, 62, 137-143, (2014)

5. N. Nurmalia, H. Ogi, M. Hirao, Jpn. J. Appl. Phys, 50, 07HC17, (2011) 
6. P.A. Petcher, S. Burrows, S. Dixon, Ultrasonics, 54, 997-1004, (2014)

7. P.A. Petcher, S. Dixon, NDT\&E Int., 74, 58-65, (2015)

8. Wei Z, Huang S, Wang, S, IEEE Sens. J., 15, 65496558, (2015)

9. Zhang Y, Huang S, Wang S, PIER M, 50, 151-160, (2016)

10. H. Ogi, E. Goda, M. Hirao, Jpn. J. Appl. Phys, 42(5S), 3020, (2003)

11. D.C. Jiles, J. Phys. D : Appl. Phys, 28, 1537-1546, (1995)

12. J.P. Joule, Phil. Mag, 30(199), 76-87, (1974)

13. N.S. Tzannes, IEEE Trans. Sonics Ultrason. 13, 3341, (1966)

14. R.B. Thompson, J. Appl. Phys, 48, 4942-4950, (1977)

15. R.B. Thompson, Appl. Phys. Lett, 34, 175-177, (1979)

16. S.H. Cho, J.S. Lee, Y.Y. Kim, Appl. Phys. Lett., 88, 224101, (2006)

17. J.S. Lee, S.H. Cho, Y.Y. Kim, Appl. Phys. Lett, 90, 054102, (2007)

18. J.S. Lee, Y.Y. Kim, S.H. Cho, Smart Mater. Struct, 18, 015009, (2009)

19. Zhou L, Yang Y, Yuan F.G, J. Vibroeng., 14(1), 280-291, (2012)

20. B. Yoo, S.M. Na, A.B. Flatau, D.J. Pines, Smart Mater. Struct, 23, 095035, (2014)

21. J.K. Lee, H.W. Kim, Y.Y. Kim, IEEE Trans. Ultrason. Ferroelectr. Freq. Control, 60, 1928-1934, (2013)

22. H.M. Seung, H.W. Kim, Y.Y. Kim, Ultrasonics, 53, 1304-1308, (2013)

23. Y. Cho, D.D. Hongerholt, J.L. Rose, IEEE Trans. Ultrason. Ferroelectr. Freq. Control, 44(1), 44-52, (1997)

24. S.K. Datta, Mech Today, 4,149-208, (1978)

25. S.K. Datta, J Appl Mech, 46, 101-106, (1979)

26. D.N. Alleyne, P. Cawley, IEEE Trans. Ultrason. Ferroelectr. Freq. Control, 39(3), 381-397, (1992)

27. T. Ghosh, T. Kundu, P. Karpur, Ultrasonics, 36(7), 791-801, (1998)

28. P.D. Wilcox, PhD Thesis. University of London, (1998)

29. M.J.S. Lowe, P. Cawley, J.Y. Kao, O. Diligent, J. Acoust. Soc. Am, 112, 2612-2622, (2002)

30. O. Diligent, T. Grahn, A. Bostrom, P. Cawley, M.J.S. Lowe, J. Acoust. Soc. Am, 112, 589-601, (2002)

31. Lu Y, Ye L, Su Z, Yang C, NDT\&E Int, 41, 59-68, (2008)

32. A. Demma, P. Cawley, M.J.S. Lowe, J. Acoust. Soc. Am., 113(4), 1880-1891, (2003)
33. P. Rajagopal, M.J.S. Lowe, J Acoust. Soc. Am, 122,1527-1538, (2007)

34. P. Rajagopal, M.J.S. Lowe, J. Acoust. Soc. Am, 124, 895-904, (2008)

35. P. Rajagopal, M.J.S. Lowe, J. Acoust. Soc. Am, 124(4), 2021-2030, (2008)

36. M. Ratassepp, M.J.S. Lowe, P. Cawley, A. Klauson, J. Acoust. Soc. Am., 124(5), 2873-2882, (2008)

37. Z. Fan, M.J.S Lowe, NDT\&E Int., 14, 124-133, (2012)

38. R. Ribichini, F. Cegla, P.B. Nagy, IEEE Trans. Ultrason. Ferroelectr. Freq. Control. 58, 2571-2581, (2011).

39. C.F. Vasile, R.B. Thompson, J. Appl. Phys., 50, 2583-2588, (1979)

40. H. Kwun, C.W. Teller, J. Acoust. Soc. Am., 96, 1202-1204, (1994)

41. J. Hettler, M. Tabatabateipour, S. Delrue, Physics Procedia, 70, 664-667, (2015)

42. Liu Z., Zhang Y, Xie M, Li A, Bin W, He C. NDT\&E Int., 97, 20-31, (2018)

43. G. Azuara, E. Barrera, M. Ruiz, EWSHM, 10-13, (2018).

44. Lu Y, Ye L, Su Z, Smart Mater. Struct, 15(3), 839$849,(2006)$ 